\title{
Type 1 and 2 Lewis antigens of Helicobacter pylori - a potential marker of the human geographical distribution
}

Lewis antigens have been found both in human gastric epithelium and in Helicobacter pylori isolates (Monteiro et al., 1998; Wirth et al., 1997). That H. pylori LPS antigens can mimic human Lewis antigens may help $H$. pylori to evade the immune response and thus enhance bacterial adherence to gastric epithelium (Monteiro et al., 2000; Sheu et al., 2003; Wirth et al., 1996). Up to now, several host Lewis antigens, such as Lewis $b\left(\operatorname{Le}^{\mathrm{b}}\right), \mathrm{Le}^{\mathrm{x}}$ and even sialyl-Le ${ }^{\mathrm{x}}$, have been shown to serve as putative adhesion receptors for bacterial blood group antigen binding adhesion (BabA), Le $\mathrm{e}^{\mathrm{x}}$ and sialyl acidbinding adhesin (SabA) of $H$. pylori isolates, respectively (Monteiro et al., 2000; Sheu et al., 2006, 2007). Such interactions between the host receptors on gastric epithelium and bacterial adhesion facilitate bacterial colonization and inflammation, and may consequently enhance virulence during long-term chronicity.

A recent cell line approach confirmed that the anti-Le ${ }^{\mathrm{x}}$ antibody significantly enhanced $H$. pylori adhesion to human gastric epithelium cells, particularly in those isolates expressing high levels of $\mathrm{Le}^{\mathrm{x}}$ antigen (Sheu et al., 2007). This study supports the novel finding of Taylor et al. (1998), who showed by electron microscopy that $\mathrm{Le}^{\mathrm{x}}$ was present on both H. pylori and gastric epithelium in adhesion pedestal formation. These data support the proposal that bacterial Lewis antigens have an impact on $H$. pylori infection outcome. Moreover, the study raised the idea checking whether Lewis antigens of $H$. pylori, other than the common type $2 \mathrm{Le}^{\mathrm{x}}$, could serve certain pathogenic roles.

H. pylori isolated from North American and European hosts predominantly expresses type 2 Lewis antigens $\left(\mathrm{Le}^{\mathrm{x}}\right.$ and Le $^{\mathrm{y}}$ epitopes) (Monteiro et al., 1998). A recent study from Chile showed that South American $H$. pylori isolates had a higher prevalence rate $(24 \%)$ of type 1 Lewis antigen (Altman et al., 2008). More strikingly, only the Le ${ }^{\mathrm{b}}$ epitope could be traced in their series. These data disproved the theory that only LPSs from Asian strains have the capacity to express Le $\mathrm{b}^{\mathrm{b}}$ structures. Furthermore, the study suggested that it would be interesting to check whether type 1 antigens, especially the $\mathrm{Le}^{\mathrm{a}}$ epitopes, were more prevalent in Asian isolates.

We checked this by studying 100 isolates in Taiwan, a nearly $100 \%$ cytotoxinassociated antigen (CagA)-positive Eastern Asian country. Our isolates carried $25 \%$ and $35 \%$ prevalence rates for type $1 \mathrm{Le}^{\mathrm{a}}$ and Le ${ }^{\mathrm{b}}$ epitopes, respectively. In North America or Europe, type 1 expression is relatively rare, and in Latin America, the type 1 (but only $\mathrm{Le}^{\mathrm{b}}$ ) prevalence rate is up to $24 \%$. However, in Asia, the prevalence rate of type 1 can be up to $35 \%$, and both $\mathrm{Le}^{\mathrm{a}}$ and Le ${ }^{\mathrm{b}}$ can exist. Accordingly, type 1 or 2 Lewis antigens of the $H$. pylori isolates should be a potential marker for identifying the geographical distribution in the worldwide human population.

Wirth et al. (1996) showed that the $\mathrm{Le}^{\mathrm{x}}$ and $\mathrm{Le}^{\mathrm{y}}$ antigens of $H$. pylori are closely correlated with CagA in $H$. pylori isolates, and thus contributed to a more severe inflammation and bacterial persistence. In the study in Chile, the majority of $H$. pylori strains examined were CagA-positive $(83.3 \%)$. Maybe due to the high CagA-positive rate, there was no significant correlation between CagA and bacterial Lewis antigens (Altman et al., 2008). Nevertheless, it is interesting that their CagA-negative isolates were uniformly shown to have no Le ${ }^{\mathrm{x}}$ antigen (Altman et al., 2008). The implication of such a finding deserves further study.

The disease outcomes of such type 1 Lewis antigen $H$. pylori infections in Chile were also studied, but maybe due to the relatively small-scale design, there was a nonsignificant equivocal correlation between type 1 infection and various clinical outcomes (Altman et al., 2008). The other weakness of the study is the lack of comparison of the histological features of patients to illustrate whether those with type 1 isolates had a heavier bacterial colonization or more severe inflammation. Based on our preliminary analysis, the isolates with coexisting type 1 and type 2 epitopes showed significantly denser $H$. pylori colonization and polymorphonuclear infiltrations than the isolates expressing type 2 epitopes only (data not shown). Thus more longitudinal data or interventional treatment trials are needed to determine the exact clinical significance of such type $1 \mathrm{H}$. pylori infections.

The reasons why Asian or Latin American people can harbour $H$. pylori isolates with more type 1 Lewis epitopes remain uncertain. The adaptation of H. pylori isolates to the host Lewis phenotype may be a good explanation. We have studied the host gastric Lewis antigen expression in 35 patients with type 1 infection in Taiwan, and significantly revealed that $\mathrm{Le}^{\mathrm{a}}$ and $\mathrm{Le}^{\mathrm{b}}$ of the infected host could concordantly match up to $90 \%$ of the type 1 Lewis epitopes of the isolates (data not shown). Accordingly, the host should have the possibility of selecting or adapting the Lewis types of $H$. pylori. It would thus be interesting to investigate whether the host Lewis antigens of the human population worldwide could show different prevalences of $\mathrm{Le}^{\mathrm{a}}$ and $\mathrm{Le}^{\mathrm{b}}$. Moreover, if Lewis antigens of $H$. pylori could serve as a virulence factor facilitating colonization or inflammation, the selection of vaccine targeting the Lewis antigens will be different for patients from different geographical populations.

Overall, the work by Altman et al. (2008) adds to previous evidence indicating the need for further study of the clinical impact of type 1 and 2 Lewis antigen $H$. pylori infections. Currently, we see that at least 
type 1 or 2 Lewis antigens of $H$. pylori could be a potential marker of the geographical distribution in the human population.

\section{Bor-Shyang Sheu ${ }^{1,2}$ and Jiunn-Jong $\mathrm{Wu}^{3}$}

${ }^{1}$ Department of Internal Medicine, National Cheng Kung University Medical Center, Tainan, Taiwan, ROC

${ }^{2}$ Institute of Clinical Medicine, National Cheng Kung University Medical Center, Tainan, Taiwan, ROC

${ }^{3}$ Institute of Basic Medical Sciences, National Cheng Kung University Medical Center, Tainan, Taiwan, ROC

Correspondence: Bor-Shyang Sheu (sheubs@mail.ncku.edu.tw)

Altman, E., Fernández, H., Chandan, V., Harrison, B. A., Schuster, M. W., Rademacher, L. O. \& Toledo, C. (2008). Analysis of
Helicobacter pylori isolates from Chile: occurrence of selective type 1 Lewis b antigen expression in lipopolysaccharide. $\mathrm{J} \mathrm{Med}$ Microbiol 57, 585-591.

Monteiro, M. A., Chan, K. H. N., Rasko, D. A., Taylor, D. E., Zheng, P. Y., Appelmelk, B. J., Wirth, H. P., Yang, M., Blaser, M. J. \& other authors (1998). Simultaneous expression of type 1 and type 2 Lewis blood group antigens by Helicobacter pylori lipopolysaccharides. J Biol Chem 273, 11533-11543.

Monteiro, M. A., Zheng, P. Y., Ho, B., Yokota, S. I., Amano, K. I., Pan, Z. J., Berg, D. E., Chan, K. H., MacLean, L. L. \& Perry, M. B. (2000).

Expression of histo-blood group antigens by lipopolysaccharides of Helicobacter pylori strains from Asian hosts: the propensity to express type 1 blood-group antigens. Glycobiology $\mathbf{1 0}$, 701-713.

Sheu, B. S., Sheu, S. M., Yang, H. B., Huang, A. H. \& Wu, J.-J. (2003). Host gastric Lewis expression determines the bacterial density of Helicobacter pylori in babA2 genopositive infection. Gut 52, 927-932.
Sheu, B. S., Odenbreit, S., Hung, K. H., Liou, C. P., Sheu, S. M., Yang, H. B. \& Wu, J. J. (2006). Interaction of host gastric sialyl-Lewis $\mathrm{X}$ and $H$. pylori SabA enhances the $H$. pylori density in patients lacking the gastric Lewis B antigen. Am J Gastroenterol 101, 36-44.

Sheu, S. M., Sheu, B. S., Yang, H. B., Li, H. Y. \& Wu, J. J. (2007). Anti-Lewis X antibody promotes Helicobacter pylori adhesion to gastric epithelial cells. Infect Immun 75, 2661-2667.

Taylor, D. E., Rasko, D. A., Sherburne, R., Ho, C. \& Jewell, L. D. (1998). Lack of correlation between Lewis antigen expression by Helicobacter pylori and gastric epithelial cells in infected patients. Gastroenterology 115, 1113-1122.

Wirth, H. P., Yang, M., Karita, M. \& Blaser, M. J. (1996). Expression of the human cell surface glycoconjugates Lewis $\mathrm{X}$ and Lewis $\mathrm{Y}$ by Helicobacter pylori isolates is related to cagA status. Infect Immun 64, 4598-4605.

Wirth, H. P., Yang, M., Peek, R. M., Jr, Tham, K. T. \& Blaser, M. J. (1997). Helicobacter pylori Lewis expression is related to the host Lewis phenotype. Gastroenterology 113, 1091-1098. 